\title{
Self-assessment questions
}

\section{An unusual rash}

\author{
Paul F Findlay, W R Primrose
}

A 74-year-old man with a history of rheumatoid disease, and a left lower lobectomy for carcinoma of lung 25 years previously was admitted as an emergency to hospital with a 3-day history of a purpuric rash. The rash was noted initially on the feet and spread proximally to involve the thighs and buttocks with involvement of the extensor surface of the upper limbs but sparing of the trunk. There were no other symptoms apart from mild colicky abdominal pain and a minor sore throat before the onset of the rash. His rheumatoid disease was quiescent at the time and there had been no recent changes in his drug therapy.

Examination confirmed the presence of a purpuric rash (figures 1 and 2) in the absence of systemic illness. Routine blood testing was normal aside from a slightly elevated urea at $7.6 \mathrm{mmol} / \mathrm{l}$ with a normal creatinine. A clotting screen was also within normal limits. The erythrocyte sedimentation rate was $26 \mathrm{~mm} / \mathrm{h}$ and a mid-stream urinalysis revealed microscopic haematuria with $1+$ red blood cells and no growth. During his period of hospitalisation he passed a melaena stool on one occasion but there was no haemodynamic compromise and all parameters remained normal.

\section{Department of Medicine for the Elderly, Woodend Hospital, Eday Road, Aberdeen AB15 6LS UK \\ P F Findlay W R Primrose}

Accepted 2 September 1998

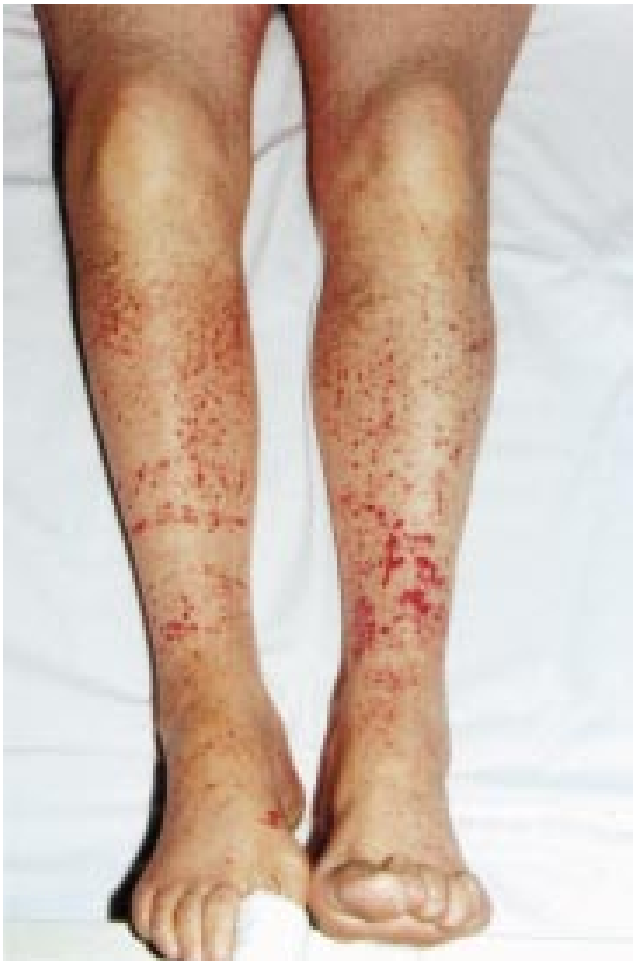

Figure 1

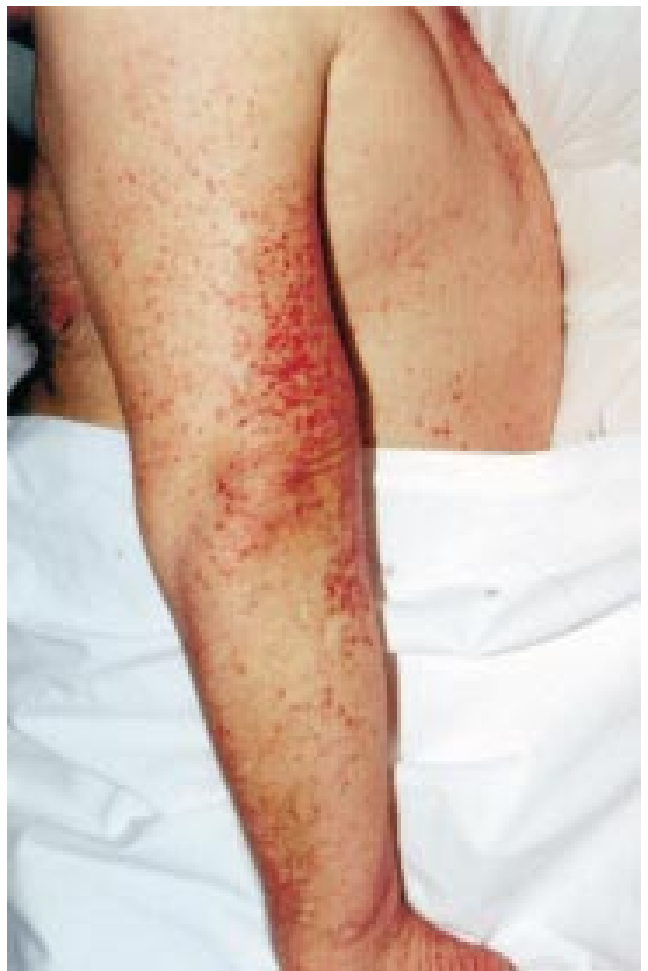

Figure 2

\section{Questions}

1 What is the probable diagnosis?

2 What complications can occur? 


\section{Answers}

QUESTION 1

Henoch-Schönlein purpura (HSP). This is an inflammatory disorder of blood vessels characterised by non-thrombocytopenic purpura, commonly distributed over the buttocks and lower extremities, arthralgia, abdominal pain and a form of glomerular nephritis. It is mediated by immune complex deposition, predominantly IgA, which affects the small blood vessels resulting in a local vasculitis. Histologically the predominant cell type is the polymorphonuclear leucocyte.

QUESTION 2

Complete recovery from this condition is the rule, but complications can occur (box).

\section{Complications of Henoch-Schönlein purpura}

- renal failure

- abdominal pain, peritonitis, bowel perforation, gastrointestinal blood loss

- pulmonary haemorrhage

\section{Discussion}

Henoch-Schönlein purpura preferentially affects children between the ages of 5 and 15 years with a slight bias towards the male gender

1 Blanco R, Martinez-Taboada VM, Rodriguez-Valverde V, Garcia-Fuentes M, Gonzalez-Gay MA. Henoch-Schonlein purpura in adulthood and childhood. Two different expressions of the same syndrome. Arthritis Rheum 1997;40:85964.

2 Miura M, Nomoto Y, Sakai H, Yamamoto O. An aged patient with Henoch-Schonlein purpura nephritis: a case patient with Henoch-Schonlein purpura nephritis: a case
report and review of the literature. Intern Med 1992;31:232rep
$(\mathrm{M}: \mathrm{F}=1.5: 1)$. In this population, which has been extensively studied, the condition is deemed to be benign and self-limiting with few complications. This disorder can, however, be expressed in adult life and until recently received little attention. A comparison of HSP in children and adults revealed that differences do exist between the two population groups. ${ }^{1}$ Cutaneous lesions were found to be the main clinical manifestation in both groups and in the adult population joint symptoms were predominant over abdominal pain in comparison with the paediatric group. Similarly, adults were preferentially subject to more renal involvement than children and often required immunosuppressive treatment. Interestingly, with regard to this particular case, preceding upper respiratory tract infection was a more frequent feature amongst children.

Case reports have appeared in the literature underlining the potential for serious sequelae of HSP in adults with nephritis, ${ }^{2}$ pulmonary haemorrhage, ${ }^{3}$ and bowel perforation ${ }^{4}$ being reported. There is also evidence for HSP as a cutaneous marker for underlying malignancy. An association with leukaemias and lymphomas, although rare, is recognised and one case report quotes the expression of HSP in tandem with the progression of a malignant breast tumour. ${ }^{5}$ Despite the different expression between the paediatric and adult forms, the final outcome in both groups is equally good.

\section{Final diagnosis}

Henoch-Schönlein purpura.

Keywords: vasculitis; Henoch-Schonlein purpura
3 Paller AS, Kelly K, Sethi R. Pulmonary haemorrhage: an often fatal complication of Henoch-Schonlein purpura. Paediatric Dermatology 1997;14:299-302.

4 Okano M, Susuki T, Takayasu H, et al. Anaphylactoid purpura with intestinal perforation : report of a case and review of the Japanese literature. Pathol Int 1994;44:303-8.

5 Maestri A, Malacarne P, Santini A. Henoch-Schonlein syndrome associated with breast cancer. A case report. Angiology 1995;46:625-7. 


\title{
Large subcutaneous nodules in lower limbs following thyroiditis
}

\author{
Atul Kakar, S P Byotra
}

A 20-year-old woman was admitted with complaints of fever and polyarthralgia since January 1996. All investigations including collagen markers were negative. The patient was treated with analgesics and showed improvement. In July she noticed a thyroid swelling with low-grade fever, and investigations showed that she was hypothyroid. Antimicrosomal antibodies were positive and antithyroglobulin was negative. Thyroid receptor antibody status was not available. Thyroid fine needle biopsy showed chronic lymphocytic thyroiditis. She was put on thyroid hormone replacement therapy.

In February 1997 she noticed multiple subcutaneous lumps in the lower limbs. She also complained of low-grade fever and arthralgia. There was no malar or discoid rash or rash elsewhere on the body. On examination, the lumps were hard, mobile, and non-tender, varying in size from 3-7 cm. She was febrile and had swelling of the small joints of both hands. Investigations showed normal blood picture, erythrocyte sedimentation rate was $130 \mathrm{~mm}$ in 1 st hour, ANA and dsDNA both were strongly positive. Anti-Sm, and antiphospholipid antibodies (IgG, IgM) were negative. Lupus anticoagulant was positive. Echocardiography showed mild pericardial effusion. Chest $\mathrm{X}$-ray was normal and X-ray of the hands did not show any erosion. Liver, renal function tests, serum calcium, phosphorus, alkaline phosphatase, parathyroid levels, creatine phosphokinase, barium swallow and pulmonary functions test were all normal. Rheumatoid factor was negative. $\mathrm{X}$-Rays of the lower limbs are shown in figures 1 and 2 .

Department of

Internal Medicine, Sir Ganga Ram Hospital, Rajinder Nagar, New Delhi-60, India A Kakar

S P Byotra

Correspondence to Dr Atul Kakar, 31 South Patel Nagar, New Delhi-110008, India

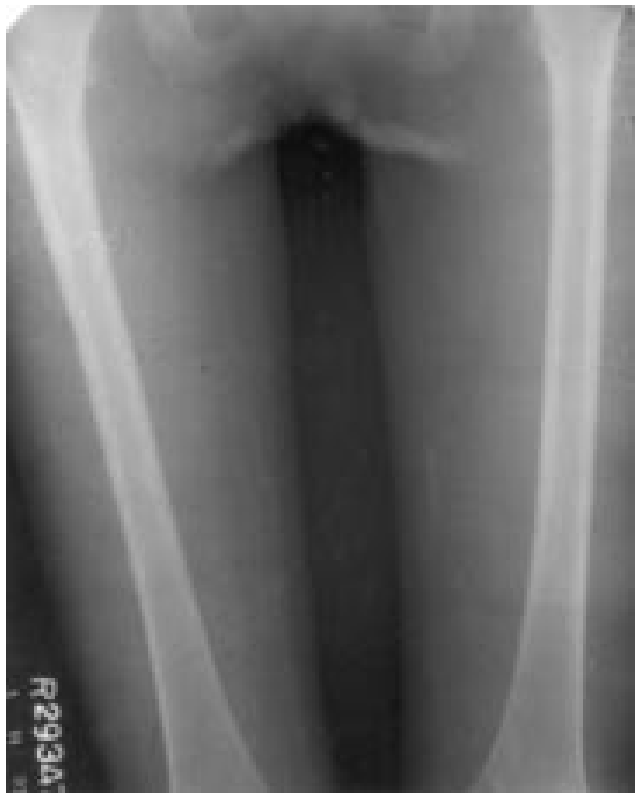

Figure 1 X-Ray of the lower limbs

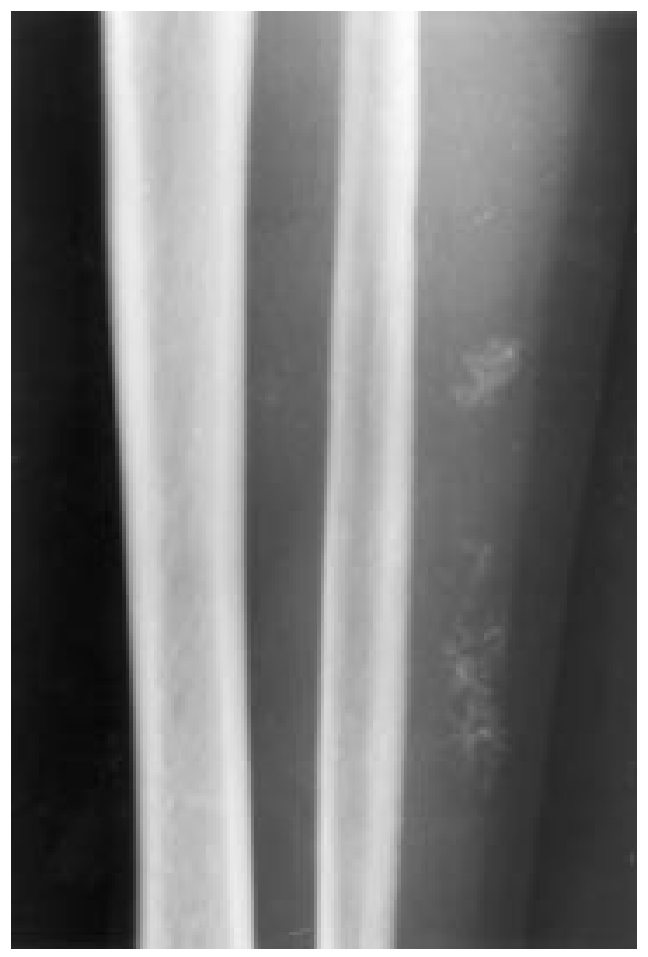

Figure 2 X-Ray of the lower limbs

\section{Questions}

1 What are the X-ray findings?

2 What is the diagnosis? 
Answers

QUESTION 1

Extensive calcification in subcutaneous tissue of lower limbs.

QUESTION 2

Calcinosis cutis in systemic lupus erythematosus (SLE). The patient fulfilled the criteria of the American Rheumatism Association for the diagnosis of SLE. Fine needle biopsy from the nodules showed calcium deposits in subcutaneous tissues and was consistent with the diagnosis of calcinosis cutis.

\section{Discussion}

Calcium deposits in the skin can occur in connective tissue disorders such as dermatomyositis and scleroderma. Although calcinosis cutis in such collagen disorders is not rare, its occurrence in cases of SLE is, ${ }^{1}$ and only 30 cases have been reported. Calcinosis cutis is extremely rare in mixed connective tissue disorders. ${ }^{2}$ Although it is common in scleroderma, it is rare in morphea and was reported in only one of 74 patients by Muller. ${ }^{3}$ The clinical examination of our patient was not suggestive of such a disorder.

Calcinosis cutis may be an incidental finding or may present as subcutaneous tumours. The calcinosis may involve a small area (circumscripta) or be diffuse (universalis). In dermatomyositis, the calcium deposits occur in shoulders and hips of young men. The deposits are usually large, ${ }^{4}$ in contrast to scleroderma in which they are small and occur in fingers of adults. ${ }^{3}$ All the above conditions are examples of dystrophic calcification and the product of serum calcium and phosphorus levels is not raised The exact mechanism of calcification in SLE is not known. The proposed mechanism involves trauma and inflammation, which denature the protein in necrotic tissue. The phosphates bind to denatured protein and act as a nidus for calcification. The other mechanism involves the increased activity of alkaline phosphatase in the local tissue by lysosomes leading to calcification. Although intralesional steroids, aluminum hydroxide, and etidronate have been shown to be useful, medical treatment is usually not successful. Surgical excision of the lesion with skin grafting has been recently shown to be beneficial. ${ }^{1}$ Our patient was unusual as she had calcinosis cutis associated with relatively mild SLE which was of shorter duration.

Our patient also had thyroid involvement, the prevalence of which is $7.5 \%$ in cases of SLE. ${ }^{5}$ This prevalence is greater than that seen in the general population. In SLE, thyroid function tests are frequently abnormal and thyroid autoantibodies may be present without disease being actually present. Thyroid disease in SLE occurs before the diagnosis of SLE is made or after stopping the immunosuppressive therapy. ${ }^{5}$

\section{Calcinosis cutis in SLE}

- rare cause of calcinosis cutis

- product of calcium and phosphorus is normal

- common site of calcinosis is lower limb

- may be associated with myopathy

- occurs with long-standing, severe disease

- calcification may be bilateral and diffuse or unilateral and localised

Box 1

Thyroid disorders in SLE

- diagnosis may be missed because of overlap of non-specific symptoms

- patients with autoimmune thyroiditis may develop SLE

- HLA-B8 DR3 occur more commonly with SLE and autoimmune thyroiditis

- both hyper- and hypothyroidism occur in SLE

- SLE patients on steroids, nonsteroidal anti-inflammatory agents, beta-blockers and dilantin would have lower T3 and T4 values while propylthiouracil used to treat thyrotoxicosis rarely causes SLE

Box 2

Dermopathy in primary hypothyroidism or chronic lymphocytic thyroiditis is exceptionally rare. ${ }^{6}$ In $15-20 \%$ patients with Graves' disease, there is dermopathy over the dorsal surface of the legs or feet called pretibial myxoedema. ${ }^{6}$ This commonly presents with ophthalmopathy and is a late manifestation of Graves' disease. Older patients are more at risk for developing this dermopathy. ${ }^{7}$ The affected area is raised, circumscribed and thickened. It has a peau d'orange-like appearance. There is a localised area of hyperpigmented and non-pitting oedema. The lesions may rarely be plaque, nodular or polypoidal. Histologically there is lymphocyte infiltration and metachromatic material is seen in the deeper layers of the dermis. $^{8}$

In calcinosis cutis the lesions may be diffuse and bilateral and commonly occur in lower limbs. ${ }^{1}$ The calcification occurs in a diffuse, linear, streaky or nodular form. Calcifications may also be plaque-like along the anterior aspect of lower limbs; they are hard on palpation. There may be associated myopathy, peripheral vascular calcification, or skin ulcerations. The skin biopsy in these patients may show extensive deposition of collagen in dermal and subcutaneous tissue with calcification.

\section{Final diagnosis}

Thyroiditis with calcinosis cutis universalis in SLE.

Keywords: thyroiditis; calcinosis cutis; systemic lupus erythematosus 
1 Cousins MAM, Jones DB, Whyte MP, Monafo WW. Surgical management of calcinosis cutis universalis in systemic lupus erythematosus. Arthritis Rheum 1997;40:570-2.

2 Baurle G, Hornstein OP. Generalized cutaneous calcinosis and mixed connective tissue disease. Dermatologica 1979; 158:257.

3 Muller SA, Brunsting LA, Winkelmann RK. Calcinosis cutis: a relationship to scleroderma. Arch Dermatol 1959;80: $15-21$.

4 Muller SA, Winkelmann RK, Brunsting LA. Calcinosis in dermatomyositis. Arch Dermatol 1959;79:669-73.
5 Miller FW, Moore GF, Weintraub BD, Steinberg AD. Prevalence of thyroid disease and abnormal thyroid function test results in patients Arthritis Rheum 1987;30:1124-9.

6 Mc Dougall R. Grave's disease. Current concepts. Med Clin North Am 1991;75:79-95.

7 Kriss JP. Pathogenesis and treatment of pretibial myxedema. Endocrinol Metab North Am 1987;16:2.

8 Johson WC, Hellwig EB. Cutaneous focal mucinosis a clinicopathological and histochemical study. Arch Dermatol 1966;93:13.

\title{
Gestational pancreatitis complicating uncontrolled diabetes mellitus
}

\author{
Shih-Yi Lin, Ya-Yu Wang, Shing-Win Shiao, Hong-Da Lin, Shih-Tzer Tsai
}

Department of

Medicine, Veterans

General

Hospital-Taipei and

National Yang Ming

University, Taipei,

Taiwan, ROC

Division of

Endocrinology and

Metabolism

S-Y Lin

S-W Shiao

H-D Lin

S-T Tsai

Division of

Gastroenterology

Y-Y Wang

Correspondence to

Dr Ya-Yu Wang, Division of

Gastroenterology,

Department of Medicine,

Veterans General

Hospital-Taipei, 201, section

2, Shih-Pai Road, Taipei

11217, Taiwan, ROC

Accepted 2 September 1998
A 36-year-old $\mathrm{G}_{5} \mathrm{P}_{2} \mathrm{AB}_{2}$ woman was admitted to our hospital due to nausea, vomiting and severe epigastric pain for one day at 24 weeks gestation. She had a history of diabetes for one year, but was not receiving any medical treatment. The patient also denied any other drug history or alcohol drinking. Physical examination revealed a blood pressure of 150/100 $\mathrm{mmHg}$, body temperature $37^{\circ} \mathrm{C}$, weight $60 \mathrm{~kg}$, and height $159 \mathrm{~cm}$. Cutaneous eruptions or xanthomas were not seen. The epigastric tenderness was severe, but without rebounding pain or muscle rigidity. Pelvic examination was unremarkable. Fundus height was $22 \mathrm{~cm}$ and foetal heart rate 140 beats/min. Laboratory studies showed a serum amylase level of $457 \mathrm{U} / 1$ (normal range $<180 \mathrm{U} / 1$ ) and lipase $1056 \mathrm{U} / 1$ (<190 U/1). Plasma sugar was $12.9 \mathrm{mmol} / 1$, triglycerides $81.8 \mathrm{mmol} / \mathrm{l}$ and total cholesterol $20.7 \mathrm{mmol} / 1$. $\mathrm{HbA}_{1 \mathrm{c}}$ was $10.2 \%$ (4-6\%), and postprandial serum C-peptide $0.2 \mathrm{nmol} / 1$. Arterial blood gasses were $\mathrm{pH}_{\mathrm{a}} 7.23, \mathrm{p}_{\mathrm{a}} \mathrm{O}_{2} 113 \mathrm{mmHg}$ at room air, $\mathrm{p}_{\mathrm{a}} \mathrm{CO}_{2} 21 \mathrm{mmHg}$, base deficit $15 \mathrm{mmol} / 1$. Blood ketone reaction was strong with 1:4 dilution. Abdominal sonography disclosed a swelling pancreas, but no evident biliary tract disease. Under a provisional diagnosis of acute pancreatitis, parenteral dextrose $(2000 \mathrm{kcal} /$ day by $50 \%$ glucose water and $0.9 \%$ glucose saline) and intravenous insulin were administered. Ketoacidosis was promptly corrected in one day and serum triglyceride was decreased to $13.5 \mathrm{~mol} / \mathrm{l}$ by the seventh day. Thereafter a low fat diet was resumed and plasma sugar was well controlled by daily subcutaneous insulin. The serum triglyceride was further lowered to $3.3 \mathrm{~mol} / 1$ after 2 weeks. The patient had a weight gain of $3 \mathrm{~kg}$ within 3 weeks of hospitalisation and finally gave birth to a healthy baby of $2600 \mathrm{~g}$ by caesarean section at the 38th week of pregnancy.

\section{Questions}

1 What is the most likely cause of acute pancreatitis in this pregnant woman?

2 What are the possible mechanisms of her extreme gestational hypertriglyceridaemia ?

3 How should her hypertriglyceridaemia be managed ? 
Answers

QUESTION 1

Gestational pancreatitis is a rare complication with a high mortality for mother and foetus, the most common cause being biliary tract disease. ${ }^{1}$ However, in women with an underlying disorder of lipid metabolism, the possibility of gestational hypertriglyceridaemic pancreatitis should never be overlooked. ${ }^{2}$ Pregnancy is normally associated with a hyperlipoproteinaemia, and may be associated with as much as a 2.5-fold increase of very low density lipoprotein triglycerides (VLDL-TG) over pregestational levels in the middle of third trimester. ${ }^{3}$ In the presence of a pre-existing lipid disorder such as familial hypertriglyceridaemia, a marked gestational hypertriglyceridaemia may occur. ${ }^{2}$ In view of the negative sonographic study of the hepatobiliary tract, denial of alcohol drinking and an extremely high plasma triglyceride level, our patient probably suffered from an acute attack of hypertriglyceridaemic pancreatitis.

QUESTION 2

Diabetes is known as a common cause of secondary lipid metabolism disorder and is often associated with increased plasma VLDL-TG levels and a marked hypertriglyceridaemia, over $2000 \mathrm{mg} / \mathrm{dl}$, may even occur in some patients. ${ }^{45}$ Recently, some authors have stated that oestrogen therapy in diabetic women may lead to an extreme hypertriglyceridaemia, particularly in an uncontrolled glycaemic state, and then possibly induce acute pancreatitis. ${ }^{6}$ However, gestational hypertriglyceridaemic pancreatitis complicating uncontrolled diabetes has rarely been reported.

It had been shown that diabetic pregnancies are associated with higher plasma triglycerides than normal ones. ${ }^{37}$ Moreover, the increased levels of plasma triglycerides has been shown to be related to diabetic control. ${ }^{7}$ The accentuated hypertriglyceridaemia may be produced by a synergistic effect of diabetes and pregnancy on VLDL synthesis. ${ }^{7}$ In our patient, who had an uncontrolled diabetes before pregnancy, this is the most probable explanation for her extreme hypertriglyceridaemia. Nevertheless, we did not perform any other investigation of genetic lipid abnormalities on our patient or her family and could not exclude the possibility of an endogenous lipid disorder, in spite of the good recovery of hypertriglyceridaemia after insulin infusion. ${ }^{5}$ Diabetic ketoacidosis, another acute metabolic complication noted in our patient, could also be associated with hypertriglyceridaemia. ${ }^{8}$ How-

1 Ramin KD, Ramin SM, Richey SD, Cunningham FG. Acute pancreatitis in pregnancy. Am $\mathcal{f}$ Obstet Gynecol 1995;173:187-91

2 De Chalain TMB, Michell WL, Berger GMB. Hyperlipidemia, pregnancy and pancreatitis. Surg Gynecol Obstet 1988;167:469-73.

3 Knopp RH, Warth MR, Charles D, et al. Lipoprotein metabolism in pregnancy, fat transport to fetus and the effects of diabetes. Biol Neonate 1986;50:297-317.

4 Howard BV, Abbott WF, Beltz WF, et al. The effect of noninsulin dependent diabetes on very low density lipoprotein and low density lipoprotein metabolism in man. Metabolism and low density

\section{Learning points}

- pre-existing hypertriglyceridaemia may be exacerbated by pregnancy and thus induce a risk of acute hypertriglyceridaemic pancreatitis

- uncontrolled diabetes may exacerbate gestational hypertriglyceridaemia

- strict glycaemic control is effective in managing gestational diabetic hypertriglyceridaemia

ever, in the reported cases, hypertriglyceridaemia over $7000 \mathrm{mg} / \mathrm{dl}$ has rarely been found and our patient's hypertriglyceridaemia could not be attributed to diabetic ketoacidosis alone. ${ }^{8}$

\section{QUESTION 3}

Management of gestational hypertriglyceridaemic pancreatitis lies in correction of lipoprotein metabolism disturbances, and maintenance of nutritional support. ${ }^{9}$ Dietary fat restriction, lipid-free parenteral nutrition or plasma exchange have all been used to achieve this. ${ }^{910}$ However, antihypertriglyceridaemic drugs, such as fibric acid derivatives should not be considered, due to the possibility of teratogenic effects. Strict glycaemic control would provide another rational therapy. ${ }^{71}$ In our patient, diabetic VLDL-TG metabolism was normalised by intensive insulin therapy, possibly mediated by decreased hepatic VLDL synthesis and enhanced lipoprotein lipase due to the effects of insulin. ${ }^{12} 13$

\section{Conclusion}

A moderate hypertriglyceridaemia due to uncontrolled diabetes may have existed in our patient before pregnancy and was markedly exacerbated by the gestation. In consequence, acute hypertriglyceridaemic pancreatitis occurred, complicated by diabetic ketoacidosis.

Pancreatitis is a rare but serious event during pregnancy. Pregnant women with inadequately controlled diabetes may be at risk of hypertriglyceridaemia. Strict diabetic control during pregnancy is warranted to prevent hypertriglyceridaemic pancreatitis and achieve an optimal outcome for mother and foetus.

\section{Final diagnosis}

Gestational hypertriglyceridaemic pancreatitis and diabetic ketoacidosis, due to uncontrolled diabetes mellitus.

Keywords: diabetes; hypertriglyceridaemia; pregnancy; pancreatitis

5 Chait A, Brunzell JD. Chylomicronemia syndrome. Adv Intern Med 1992;37:249-73.

6 Glueck CJ, Lang J, Hamer T, Tracy T. Severe hypertriglyceridemia and pancreatitis when estrogen replacement therapy is given to hypertriglyceridemic women. $7 \mathrm{Lab}$ Clin Med 1994;123:59-64.

7 Hollingsworth DR. Alterations of maternal metabolism in normal and diabetic pregnancies: differences in insulindependent, non-insulin dependent, and gestational diabetes. Am f Obstet Gynecol 1983;146:417-29.

8 Fulop M, Eder H. Severe hypertriglyceridemia in diabetic ketosis. Am f Med Sci 1990;300:361-5. 
9 Sanderson SL, Iverius PH, Wilson DE. Successful hyperlipemic pregnancy. $7 A M A$ 1991;265:1858-60.

10 Weinber RB, Si. demic pancreatitis in pregnancy with total parenteral nutrition. Gastroenterology 1982;83:1300-5.

11 Reece EA, Homoko C, Wiznitzer A. Metabolic changes in diabetic and nondiabetic subjects during pregnancy. Obstet Gynecol Surv 1994;49:64-1.
12 Dunn FL, Raskin P, Bilheimer D, Grundy SM. The effect of diabetic control on very low density lipoprotein-triglyceride metabolism in patients with type II diabetes mellitus and marked hypertriglyceridemia. Metabolism 1984;33:117-23.

13 Howard BV, Howard WJ. Dyslipidemia in non-insulindependent diabetes. Endocr Rev 1994;15:263-74.

\title{
A young woman with fluctuating hypo- and hyperthyroidism
}

\author{
A Bhattacharyya, K G Chan, D J Tymms
}

A 30-year-old woman presented for the first time in August 1990 with signs and symptoms of hypothyroidism: weight gain, fatigue, and muscle aches. She had a small goitre, but no ophthalmopathy or dermopathy. She was a non-smoker and mother of two (last childbirth 2 years previously). There was no history of thyroid disease or drug consumption. Thyroid function tests confirmed the diagnosis of primary hypothyroidism: thyroid-stimulating hormone (TSH) $74 \mathrm{mU} / 1$ (reference range $0.4-5 \mathrm{mU} / \mathrm{l})$, free thyroxine $\left(\mathrm{FT}_{4}\right) 2.4 \mathrm{nmol} / \mathrm{l}(9-24)$. Thyroid microsomal antibody (TMA) and antithyroglobulin antibody were positive (one in 256000 and 1600, respectively). Replacement therapy with thyroxine was started (100 $\mu \mathrm{g}$ daily). Over the next 6 months her dose requirement for thyroxine was reduced to $50 \mu \mathrm{g}$ daily and the drug was stopped altogether one year after the initial diagnosis while she remained clinically and biochemically euthyroid. In July 1997, she developed symptoms and signs of thyrotoxicosis: weight loss, palpitations, and increased sweating. The diagnosis of thyrotoxicosis was confirmed (TSH undetectable, tri-iodothyronine $\left(\mathrm{T}_{3}\right) 2.5 \mathrm{nmol} / 1(0.7-2.1)$, and $\left.\mathrm{FT}_{4} 29 \mathrm{nmol} / \mathrm{l}\right)$. No treatment was given. While on follow-up over the next 2 months her symptoms subsided and she gained weight; thyroid function confirmed hypothyroidism (TSH 84 mU/1, $\mathrm{FT}_{4} 8.8 \mathrm{nmol} / \mathrm{l}$ ). In November 1997, although symptomatically she remained well, thyroid function tests showed thyrotoxicosis (TSH undetectable, $\mathrm{FT}_{4} 30 \mathrm{nmol} / \mathrm{l}$ ) but she has remained euthyroid on follow-up. Her TMA titre remains the same.

\section{Department of Medicine (Division of Endocrinology), Royal Albert Edward Infirmary, Wigan, UK A Bhattacharyya K G Chan \\ D J Tymms \\ Correspondence to Dr DJ Tymms, The Hawthorns, Royal Albert Edward Infirmary, Wigan Lane, Wigan WN1 2NN, UK}

Accepted 4 August 1998

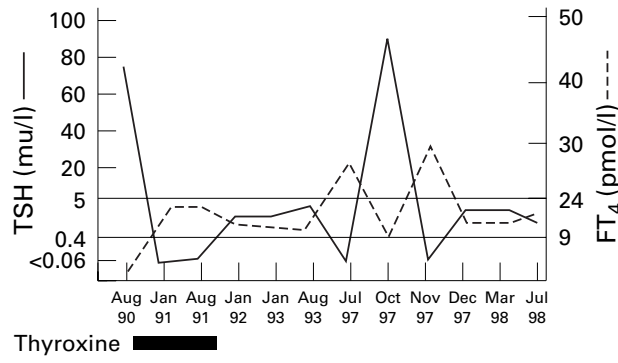

\section{Questions}

1 What is the diagnosis?

2 Why is the thyroid function fluctuating?

3 How would you treat this patient?

Figure Fluctuation of thyroid function during the follow-up period. 


\section{Answers}

QUESTION 1

Autoimmune thyroid disease with fluctuating hypo- and hyperthyroidism.

QUESTION 2

The fluctuations are due to the presence of blocking and stimulating antibodies.

QUESTION 3

There are three possible ways of treating such a patient. The patient must be fully informed about the nature and likelihood outcome of each treatment option:

- following up with serial checks of thyroid function and treatment according to the severity and nature of the thyroid dysfunction

- blocking-replacement therapy with thyroxine and carbimazole or propylthiouracil

- radioiodine treatment with appropriate follow-up.

\section{Discussion}

Fluctuating thyroid function can be defined as a spontaneous shift of functional thyroid state from hyperthyroidism to hypothyroidism or vice versa. In autoimmune thyroid disease, thyroid function can fluctuate. Thyroidstimulating immunoglobulin (measured by the capacity of serum immunoglobulin to stimulate cAMP production in cultured human thyroid cells) stimulates the thyroid gland while blocking antibodies (measured by the degree of inhibition of TSH-stimulated CAMP production of cultured human thyroid cells by serum immunoglobulin) do the opposite. At a particular point, it is the balance between the two antibodies that decides the functional thyroid state. Although not common, similar cases have been reported for hyperthyroidism to hypothyroidism ${ }^{1}$ and vice versa. ${ }^{23}$ The end point of all reported cases was hypothyroidism, either spontaneously or as a result of treatment, but our patient remained in a euthyroid state without intervention.

Thyrotoxicosis factitia should be considered in the differential diagnosis. Clinically, the presence of goitre, ophthalmopathy or dermopathy is against the diagnosis. In thyrotoxicosis factitia, radioactive iodine uptake of the

1. Kasagi K, Hidaka A, Endo K, et al. Fluctuating thyroid function depending on the balance between stimulating and blocking types of TSH receptor antibodies: a case report. Thyroid 1993:3:315-8.

2 Takeda K, Takamatsu J, Kasagi K, et al. Development of hyperthyroidism following primary hypothyroidism: a case report with changes in thyroid related antibodies. Clin Endocrinol 1988;28:341-4.

3 Kraiem Z, Baron E, Kahana L, Sadeh O, Sheinfeld M. Changes in stimulating and blocking antibodies in a patient undergoing three cycles of transition from hypo to hyperthyroidism and back to hypothyroidism. Clin Endocrinol 1992;36:211-4 thyroid gland will be negligible, and serum thyroglobulin and antithyroid antibodies will be undetectable. Also, on biochemical testing, serum $\mathrm{T}_{3}$ is likely to be normal or low with high serum thyroxine and suppressed TSH. The presence of goitre, high serum $\mathrm{T}_{3}$ and high titre of antibodies clearly argue against such a diagnosis in our patient.

The next question is whether or not we can predict such fluctuating behaviour of the thyroid gland. No clear answer is available. Progressive destruction of the thyroid gland or production of TSH receptor blocking antibodies may be responsible for the development of a hypothyroid state in patients with Graves' hyperthyroidism. ${ }^{4}$ One can assume that blocking antibody plays a greater role than structural damage of the gland in such cases, otherwise the chance of development of a hyperthyroid phase would be very low. On the other hand, hypothyroid patients with radioiodine uptake are likely to remit, ${ }^{5}$ in contrast to those with normal or low uptake. Finally, there may be some yet unidentified factors other than stimulating and blocking antibodies, which are responsible for such fluctuations. It is interesting to note that the very high level of serum TSH at diagnosis and follow-up did not predict the outcome in our patient. Thus, a very high TSH or a very low $\mathrm{FT}_{4}$ does not necessarily mean the patient will have permanent hypothyroidism.

We believe that radioactive iodine is the best way to treat the hyperthyroid phase, with subsequent observation. Our patient's hyperthyroid phase was short lasting and of less magnitude but such a treatment option will be considered in future, if necessary. In the hypothyroid stage, the only option remains replacement with thyroxine to keep the patient euthyroid. Surgery is an alternative option in the hyperthyroid patient after stabilisation with a blocking-replacement regimen.

\section{Final diagnosis}

Autoimmune thyroid disease with fluctuating thyroid function as a result of stimulating and blocking antibodies.

Keywords: hyperthyroidism; hypothyroidism; autoimmune thyroid disorder
4 Tamai H, Kasagi K, Hara T, et al. Follow up study of thyroid stimulating blocking antibodies in hypothyroid patients. Clin Endocrinol 1990;33:699-707.

5 Okamura K, Sato K, Ikenoue H, et al. Re-evaluation of the thyroidal radioiodine uptake test, with special reference to reversible primary hypothyroidism with elevated thyroid radioiodine re-uptake. F Clin Endocrinol Metab 1988;67: $720-6$. 


\title{
A newborn with watery diarrhoea
}

\author{
Mohamed Zaki, Khalil B Gallad, Dina G Ramadan, Sameer Ogila
}

Farwania Hospital, Kuwait

Department of

Paediatrics

M Zaki

S Ogila

Department of

Obstetrics and

Gynaecology

K B Gallad

Department of Paediatrics, Sabah Hospital, Kuwait

D G Ramadan

Correspondence to $\mathrm{Dr}$ Mohamed Zaki, PO Box 25850 Safat, Code 13119, Kuwait

Accepted 2 September 1998

A 35-year-old woman was referred to hospital at 32 weeks gestation because her uterus was large for date. Ultrasound examination done at that time is shown in the figure. At 34 weeks gestation, she gave birth to a baby boy weighing $2.2 \mathrm{~kg}$, who had significant abdominal distension at birth. There was no vomiting. He did not pass meconium but after per rectum examination a large amount of light yellow watery stool was passed, followed by some relief of his abdominal distension. He continued to pass this type of stool. Blood laboratory tests at the age of 2 weeks showed a serum sodium of $131 \mathrm{mmol} / 1$, potassium $3.1 \mathrm{mmol} / 1$, chloride $91 \mathrm{mmol} / 1$ and bicarbonate 31 $\mathrm{mmol} / \mathrm{l}$. The family had an elder son who had had chronic diarrhoea since birth.

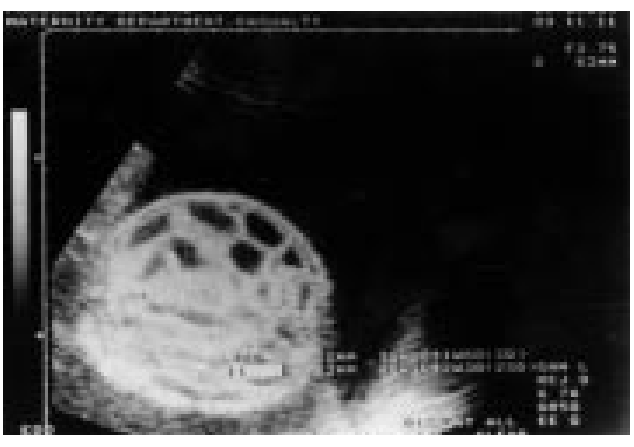

Figure

\section{Questions}

1 What does the sonogram show?

2 What is the most likely diagnosis?

3 How can the diagnosis be confirmed? 
Answers

QUESTION 1

Antenatal ultrasound at 32 weeks gestation showing marked dilatation of foetal intestinal loops and polyhydramnios.

QUESTION 2

The most likely diagnosis is congenital chloride diarrhoea (CCD). The diagnosis was suspected on the basis of maternal polyhydramnios, dilated foetal intestinal loops, premature delivery, absence of meconium, passage of large amount of watery stool since birth, blood tests showing hypochloraemic alkalosis, and the presence of a sibling with chronic diarrhoea since birth. The diagnosis was confirmed in this sibling to be CCD.

QUESTION 3

Confirmation of the diagnosis requires the measurement of both stool and urine electrolytes. In CCD there is a significant increased chloride concentration in stool (exceeding the sum of sodium and potassium) with no chloride in the urine.

\section{Discussion}

CCD is a rare autosomal recessive disorder with a worldwide occurrence, although most cases have been reported from Finland and the Middle East. ${ }^{1-3}$ In certain areas in the Arabian Peninsula there is an incidence of $1: 5500 .^{3}$ Recently, the CCD gene has been accurately located on chromosome $7 .^{4}$

In CCD, there is absence or impairment of active chloride/bicarbonate exchange in both the ileum and colon. The defective chloride absorption leads to osmotic diarrhoea. This malabsorption also explains the metabolic abnormalities seen in these patients.

The clinical presentation varies with the age of the child. During foetal life, significant maternal polyhydramnios occurs as a result of the intrauterine foetal diarrhoea. Antenatal ultrasound demonstrates significant dilatation of the foetal intestinal loops (figure).

Almost all patients with CCD are born prematurely, possibly as a result of the intrauterine diarrhoea. Postnatally, these children exhibit significant abdominal distension due to fluid accumulating in their intestine. There is no passage of meconium at birth and stools are profuse and watery. The diarrhoea may passed unnoticed because the fluid is thought to be urine and this can cause delay in diagnosis. Some infants survive for several months without diagnosis. These children fail to grow and their developmental milestones are delayed. Any intercurrent infection, particularly gastroenteritis, can disturb further the delicate water and electrolyte balance and can lead to their demise. In the early neonatal period, the blood biochemistry is within normal limits with occasional acidosis, but after a few weeks, they show the characteristic laboratory finding of

\section{Summary points}

- CCD is rare inherited disorder mostly seen in communities with a high percentage of consanguineous marriages

- antenatal ultrasound by an experienced operator can suggest the diagnosis

- postnatally, differentiation from intestinal atresia is important to avoid unnecessary surgery

- the characteristic metabolic abnormality of hypochloraemic alkalosis is not present from birth but takes several weeks to develop

- life-long supplementation with sodium and potassium chloride solution is required to correct the metabolic abnormality, although this therapy will not change the character of the stool

- with early and adequate therapy these children can achieve normal growth and development

hypochloraemic hypokalaemic metabolic alkalosis. Other medical conditions can show similar metabolic abnormalities; especially important are cystic fibrosis and Bartter syndrome. The high faecal chloride $(>100 \mathrm{mmol} / \mathrm{l})$, exceeding the sum of faecal sodium and potassium and a chloride-free urine will confirm the diagnosis. The presence of other siblings with CCD, as in the case presented, will allow early identification of similar cases in the family.

Because of the antenatal ultrasonographic findings, the postnatal abdominal distension and the absence of passage of meconium, other surgical conditions may be considered before the correct diagnosis is made. These conditions include intestinal atresia, meconium ileus and aganglionosis. Some newborn infants with CCD are subjected to unnecessary laparotomies because of misdiagnosis as one of the above acute surgical conditions. ${ }^{5}$ The diagnosis of CCD cannot be verified by amniocentesis since the electrolyte concentration in amniotic fluid is normal.

Since the intestinal defect cannot be corrected, life-long treatment with both sodium and potassium chloride solutions is required. The rationale behind this therapy is the normal jejunal absorption of these electrolytes. Such treatment will not correct the diarrhoea but will prevent its secondary effects. Chronic hypovolaemia and hypokalaemia will result in renal tubular dysfunction in some of these children. Children with CCD are at increased risk of developing volvulus postnatally, a complication that may occur at any age from 2 days to 15 years. ${ }^{6}$ If the diagnosis is made early and treatment is given adequately, children with CCD can achieve normal physical growth and mental development.

\section{Final diagnosis}

Congenital chloride diarrhoea.

Keywords: chloride diarrhoea; newborn; diarrhoea; intestinal atresia 
1 Holmberg C, Perheentupa J, Launiala K, Hallman N. ConHolmberg C, Perheentupa J, Launiala K, Hallman N. Congenital chloride diarrhoea; clinical analysis

2 Lubani MM, Doudin KI, Sharda DC, et al. Congenital chloride diarrhoea in Kuwaiti children. Eur $\mathcal{F}$ Pediatr 1989;148:333-6.

3 Kagalwalla AF. Congenital chloride diarrhoea; a study in arab children. $\mathcal{F}$ Clin Gastrenterol 1994;19:36-40.
4 Hoglund P, Sistonen P, Norio R, et al. Fine mapping of congenital chloride diarrhoea gene by linkage disequilibrium. Am 7 Hum Genet 1995;57:95-102

5 Lundkvist K, Anneen G, Esscher T, et al. Surgical implication of congenital chloride diarrhoea. Z Kinderchir 1983;38:217-9. 6 Kirkinen P, Jouppila P. Prenatal ultrasonic findings in congenital chloride diarrhoea. Prenat Diagnose 1984;4:457-

\title{
An unusual cause of leg ulceration
}

\author{
D Banerjee, J Grey, K G Harding
}

A 46-year-old man was referred with a 2-year history of non-healing ulcers on both lower limbs. He had suffered two episodes of deep vein thrombosis, at the ages of 7 and 28 years. He was subsequently admitted to hospital for treatment of pulmonary embolism at the age of 30 . A year earlier, he had suffered an uncomplicated inferior wall myocardial infarction. On examination, he had multiple superficial ulcers on both his legs in the gaiter areas (figure). The surrounding skin showed signs of advanced venous insufficiency in the form of venous eczema, lipodermatosclerosis and pigmentation. Duplex imaging of his venous system revealed extensive bilateral post-phlebitic changes. His only medication was warfarin, which he had been advised to take lifelong. His 18-year-old son was also diagnosed to have a deep vein thrombosis and subsequent investigations revealed two of his three children to be suffering from the same condition.

\section{Wound Healing Research Unit, University of Wales College of Medicine, Cardiff CF4 4XN, Wales, UK \\ D Banerjee J Grey \\ K G Harding}

Accepted 2 September 1998

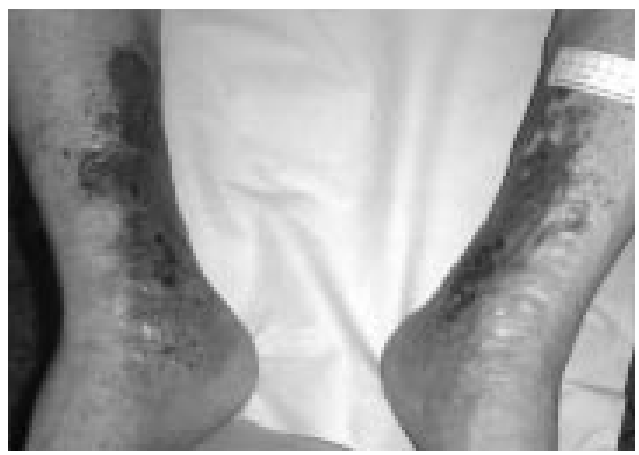

\section{Questions}

1 What is the underlying condition?

2 How is this condition managed?

3 How should the ulcers be treated?

Figure The patient's legs 
Answers

QUESTION 1

This patient was found to have hereditary protein $\mathrm{S}$ deficiency (PSD). It is an autosomal dominant disorder, associated with an increased risk for developing thrombosis in heterozygotes ${ }^{1}$; hence the higher prevalence among ethnic groups practising consanguinity. The thrombotic episodes are rare before the age of 15 but it has been reported in a 10-yearold boy. ${ }^{2}$ The acquired form of PSD can be due to various disorders including advanced malignancy, chicken pox and other viral infections (eg, HTLV-I). ${ }^{3}$

Among the known plasma protein deficiencies associated with venous thrombosis, protein $\mathrm{S}$ and protein $\mathrm{C}$ deficiencies are the commonest. ${ }^{4}$ Protein $\mathrm{S}$ is the vitamin K-dependent cofactor of activated protein $\mathrm{C}$ (APC) which functions as a potent anticoagulant by degrading activated factors $\mathrm{V}$ and VIII in a $\mathrm{Ca}^{2+}$ and phospholipid-dependent reaction. ${ }^{5}$ Protein $S$ exists as two forms in plasma, either free and functionally active or complexed with $\mathrm{C} 4 \mathrm{~b}$-binding protein and inactive. Inherited resistance to APC was recently discovered as a cause of familial thrombophilia and is now known to be the most common genetic risk factor for venous thrombosis. APC resistance is usually associated with a single point mutation in the factor $\mathrm{V}$ gene, which results in substitution of arginine at position 506 by glutamine. The mutation renders factor Va partially resistant to degradation by APC, which leads to a hypercoagulable state and increased risk of venous thrombosis. The previously known inherited deficiencies of antithrombin, protein S, or protein $\mathrm{C}$, are, in western societies, together found in less than $10-15 \%$ of thrombosis patients, whereas APC resistance is present in $20-60 \%$ of the patients. ${ }^{6}$

QUESTION 2

In young patients with recurrent venous thromboembolism in the absence of obvious predisposing factors, it is important to exclude inherited plasma protein deficiencies of protein $\mathrm{S}$, protein $\mathrm{C}$, antithrombin III, plasminogen and fibrinogen.

1 Petersen EJ, Allaart RC, Meuwissen OJ. A Dutch family with hereditary protein S deficiency. Neth $f$ Med 1989;34:243-50

2 Zoller B, He X, Dahlback B. Homozygous APC-resistance combined with inherited type I protein S deficiency in a young boy with severe thrombotic disease. Thromb Haemostasis 1995;73:743-5.

3 Schwartz J, Gonzalez 3, Rosenberg R, et al. Cutaneous T-cell lymphoma, tropical spastic paraparesis, cerebral vasculitis, and protein S deficiency in a patient with vasculitis, and protein S deficiency in

4 Gladson CL, Scharrer I, Hach V, Beck KH, Griffin JH. The frequency of type I heterozygous protein $\mathrm{S}$ and protein $\mathrm{C}$ deficiency in 141 unrelated young patients with venous thrombosis. Thromb Haemostasis 1988;59:18-22.

\section{Learning points}

- among the known plasma protein deficiencies associated with venous thrombosis, protein $\mathrm{S}$ and protein $\mathrm{C}$ deficiencies are the commonest

- protein $\mathrm{S}$ deficiency is an autosomal dominant disorder of the clotting system resulting in a hypercoagulable state; an acquired form of the condition can result from various systemic disorders

- protein S deficiency should be suspected in any young patient suffering from deep vein thrombosis or other thrombotic episode and a thrombophilia screen should be performed.

- a thorough family history should be obtained and close family members screened

- recurrent venous thromboses are common in such patients, starting at a very young age, and current practice advocates life-long anticoagulant prophylaxis

- in patients who develop post-phlebitic changes in their limbs, including ulceration, treatment comprises compression and topical wound care

Treatment is predominantly prophylactic, in the form of long-term oral anticoagulation with warfarin. Where its use is contraindicated, for example, in pregnancy, or in the event of warfarin-induced skin reactions, heparin is a suitable alternative. At present, there is lack of consensus regarding the duration of treatment. Current practice seems to favour life-long warfarin therapy.

\section{QUESTION 3}

The ulcers are initially treated with topical iodine-impregnated dressing and graduated compression therapy. Venous eczema is treated effectively with topical steroid preparations, usually over 10-12 weeks. After the first few weeks of healing, the compression therapy should be changed to a four-layer bandage system. ${ }^{7}$ The ulcers in this patient had healed satisfactorily by the end of 12 weeks of treatment.

\section{Final diagnosis}

Protein S deficiency.

Keywords: protein S deficiency; venous thrombosis

5 Wiesel ML, Charmantier JL, Freyssinet JM, Grunebaum L, Schuhler S, Cazenave JP. Screening of protein S deficiency using a functional assay in patients with venous and arterial thrombosis. Thromb Res 1990;58:461-8.

6 Dahlback B, Zoller B, Hillarp A. Inherited resistance to activated protein $C$ caused by presence of the Fv:Q506 allele as a basis of venous thrombosis. Haemostasis 1996;26(suppl 4):301-14.

7 Nelson EA, Harper DR, Ruckley CV, et al. A randomised trial of single-layer and multi-layer bandages in the treatment of chronic venous ulceration. Phlebology 1995; (suppl 1):915-6. 


\title{
A new diabetic patient with an abdominal mass
}

\author{
Boji Varghese, W P Stephens
}

A 47-year-old woman was referred to the new patient diabetic clinic with typical symptoms of hyperglycaemia. On routine abdominal examination, a mass was found on the left side of the abdomen which was ballottable and bimanually palpable. There was a history of 'benign retinal tumour' several years ago which did not need active treatment. Further enquiry revealed this to have been a retinal angioma. Routine laboratory tests on blood and urine were unremarkable except for findings of diabetes mellitus. She went on to have ultrasound scan followed by computed tomography (CT) of the abdomen (figure).

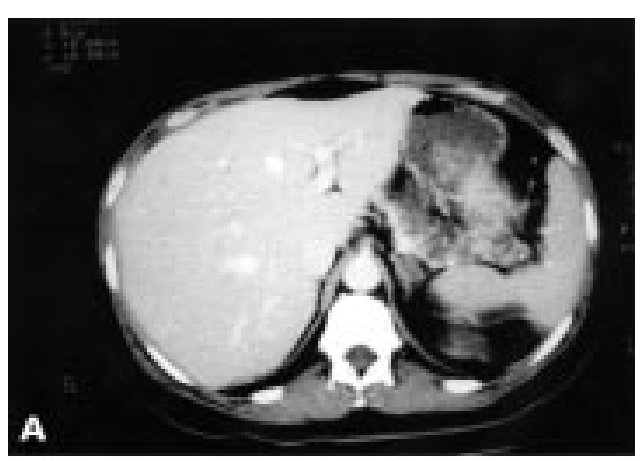

\section{Questions}

1 What is the main abnormality seen on the CT scan and what is the radiological diagnosis?

2 What other abnormalities are present on the CT scan?

3 What condition does the patient have?

4 What other abnormalities may occur in this condition?

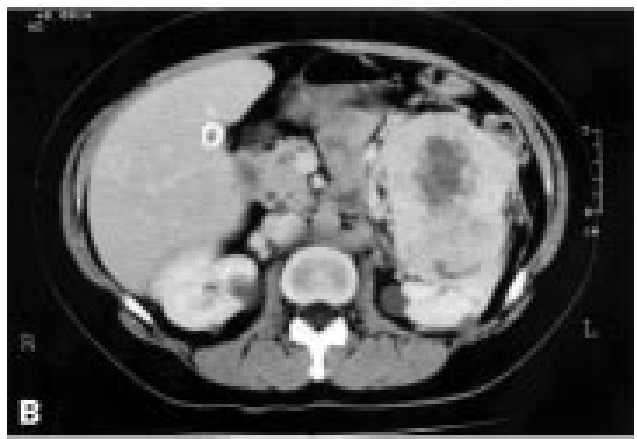

Department of

Medicine, Trafford

General Hospital,

Moorside Road,

Davyhulme,

Manchester M41 5SL,

UK

B Varghese

W P Stephens

Correspondence to

Dr B Varghese,

13 Reeves Court,

Canterbury Gardens,

Salford, Manchester

M5 2AE, UK

Accepted 2 September 1998

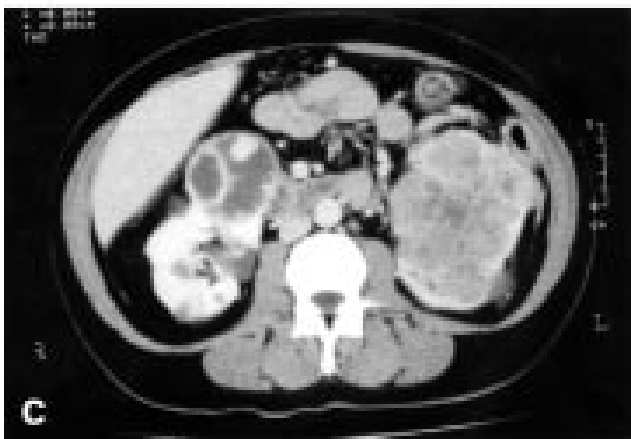

Figure $\mathrm{CT}$ scan of the abdomen 


\section{Answers}

QUESTION 1

There is a large solid tumour arising anteriorly from the lower half of the left kidney, with a necrotic centre. There are two solid tumours of the right kidney, with the anterior tumour predominantly necrotic. These appearances are consistent with bilateral hypernephromas.

QUESTION 2

There are multiple bilateral simple renal cysts, multiple pancreatic cysts and a small left adrenal mass.

QUESTION 3

The combination of retinal angioma and bilateral hypernephromas indicates the diagnosis of von Hippel-Lindau syndrome.

QUESTION 4

von Hippel-Lindau syndrome is a rare genetic disorder with multi-organ involvement. The most common features are retinal angiomatosis $(51 \%)$, haemangioblastoma of the central nervous system (46\%), and renal lesions $(33 \%) .{ }^{1}$ The pancreatic features of the condition include pancreatic cysts, endocrine deficiency, ${ }^{2}$ exocrine deficiency, ${ }^{3}$ metastatic islet cell tumour, ${ }^{4}$ and acute pancreatitis. ${ }^{5}$ The other features are phaeochromocytomas $(24 \%)$, epididymal cystadenomas and, rarely, carcinoid tumour of the bile duct ${ }^{6}$ and deafness due to bilateral endolymphatic sac tumours. ${ }^{7}$

\section{Discussion}

von Hippel-Lindau syndrome is an autosomal dominant inherited disease with a prevalence of 1:40 000. ${ }^{1}$ The von Hippel-Lindau (VHL) gene has been localised and cloned at human chromosome 3p25-p26. ${ }^{8}$ The VHL gene product has been found to suppress growth of renal cell carcinoma lines in vitro. ${ }^{9}$ The association of von Hippel-Lindau disease with renal cell carcinoma may be explained either by mutation of or loss of both alleles of the VHL gene or by disturbance in the function of the VHL gene product. $^{9} 10$

Although commonly referred to as cerebelloretinal haemangioblastomatosis, there are other significant clinical associations like renal cell carcinoma and phaeochromocytoma. Our

1 Neumann HPH, Wiestler OD. Clustering of features of von Hippel-Lindau syndrome: evidence for a complex genetic locus. Lancet 1991;337:1052-4.

2 Thompson RK, Peters JI, Sirinek KR. von Hippel-Lindau syndrome presenting as pancreatic endocrine insufficiency: a case report. Surgery 1989;105:598-604.

3 Fishman RS, Bartholomew LG. Severe pancreatic involveFishman RS, Bartholomew LG. Severe pancreatic involve-
ment in three generations of von Hippel-Lindau disease. ment in three generations of
Mayo Clin Proc 1979;54:329.

Mayo Clin Proc 1979;54:329.
4 Cornish D, Pont A, Minor D. Metastatic islet cell tumour in von Hippel-Lindau disease. Am f Med 1984;77:147-9.

5 Tener S, Roston A, Lichtenstein D, Sica G. von HippelLindau disease complicated by acute pancreatitis and Evan's syndrome. Int $\mathcal{F}$ Pancreatol 1995; 18:271-5.

6 Fellows IW, Leach IH, Smith PG. Carcinoid tumour of the common bile duct - a novel complication of von HippelLindau syndrome. Gut, 1990;31:728-9.

7 Kempermann G, Neumann HPH, Scheremet R. Deafness due to bilateral endolymphatic sac tumours in a case of von patient was known to have retinal angiomas. Subsequent CT scan of the brain did not show evidence of intracranial haemangioblastoma. Both of the renal tumours were biopsied under CT guidance and histological examination showed adenocarcinoma. Urinary normetadrenaline screening for phaeochromocytoma was negative.

Although pancreatic cysts are common, pancreatic endocrine or exocrine deficiency is uncommon. Our patient presented with symptoms of diabetes mellitus which was treated by dietary means. There was no clinical suggestion of steatorrhoea. Central nervous system lesions in von Hippel-Lindau disease present as haemangioblastomas and metastatic renal carcinomas. The haemangioblastomas are mainly intracranial ( $74 \%$ ), the rest being located in the spinal cord. ${ }^{11}$ Retinal angiomatosis present as both capillary and cavernous haemangiomas. The renal lesions include renal angiomas, renal cysts and renal carcinomas. Renal carcinomas are frequently bilateral and, if not diagnosed early, tend to metastasize. Our patient went on to have bilateral nephrectomy and renal replacement therapy with chronic ambulatory peritoneal dialysis. Parenchymal sparing surgery is sometimes used in selected patients with renal carcinoma. ${ }^{12}$

The initial presentation of von HippelLindau syndrome could be to a medical or surgical speciality but more awareness of the syndrome and its associations will lead to early diagnosis. The finding of retinal angiomas or CNS haemangioblastomas should lead to radiological assessment of the kidneys. Screening of first degree relatives will help in early identification of affected individuals.

Formal referral to a medical genetics service will help detailed screening of the patients and their relatives. Medical genetics clinics normally maintain computer registers of such families and are best placed to ensure their adequate prolonged follow-up.

\section{Final diagnosis}

von Hippel-Lindau syndrome.

Keywords: von Hippel-Lindau syndrome; renal cell carcinoma; diabetes mellitus

We are grateful to Dr P Norburn for providing CT films.

Hippel-Lindau syndrome. $f$ Neurol Neurosurg Psychiatry 1996;61:318-20.

8 Latif F, Tory K, Gnarra J, et al. Identification of the von Hippel-Lindau disease tumour suppressor gene. Science 1993;260:1317-20

9 Chen F, Kishida T, Duh FM, et al. Suppression of growth of renal carcinoma cells by the von Hippel-Lindau tumour suppressor gene. Cancer Res 1995;55:4804-7.

10 Decker HJ, Weidt EJ, Brieger J. The von Hippel-Lindau tumour suppressor gene. A rare and intriguing disease opening new insight into basic mechanisms of carcinogenesis. Cancer Genet Cytogenet 1997;93:74-83.

11 Neumann HPH, Eggert HR, Sheremet R, et al. Central nervous system lesions in von Hippel-Lindau syndrome. $\mathcal{F}$ Neurol Neurosurg Psychiatry 1992;55:898-901.

12 Walther MM, Choyke PL, Weiss G, et al. Parenchymal sparing surgery in patients with hereditary renal cell carcinoma. f Urol 1995;153:913-6. 


\title{
Fever and dyspnoea in a young man with a rash
}

\author{
Michelle N Dizon, Glenn Matfin
}

A 36-year-old male smoker presented with a 7-day history of fever, chills and malaise. He admitted to increasing dyspnoea over the past 2 days. There was no history of cough, nausea or vomiting. The patient had developed crops of erythematous pruritic vesicles on his face, trunk, and extremities 5 days prior to admission. He admitted to being exposed to a child with a similar rash, and his own three sons had developed a similar rash.

On examination he appeared to be severely ill. Skin examination revealed multiple vesicular lesions with crusting, mostly on his chest, back, and extremities. An arterial blood gas revealed $\mathrm{pH}$ $7.42, \mathrm{pO}_{2} 6.63 \mathrm{kPa}, \mathrm{pCO}_{2} 4.03 \mathrm{kPa}$, and $\mathrm{O}_{2}$ saturation of $87 \%$ on room air. He was febrile with a temperature of $39^{\circ} \mathrm{C}$. His lungs were clear to auscultation bilaterally. Routine blood tests were normal except for neutrophil leucocytosis (white blood cells of $10 \times 10^{9} / 1$ with $89 \%$ neutrophils, $3.3 \%$ lymphocytes, $6.4 \%$ monocytes, $0.1 \%$ eosinophil, and $0.8 \%$ basophil). His chest X-ray is shown in the figure.

\section{Department of Internal Medicine, University of South Florida, College of Medicine, Tampa, FL 33612, USA \\ M N Dizon \\ G Matfin \\ Correspondence to Michelle N Dizon, MD, Medical Service III, 13000 Bruce B Downs Blvd, Tampa, FL 33612, USA}

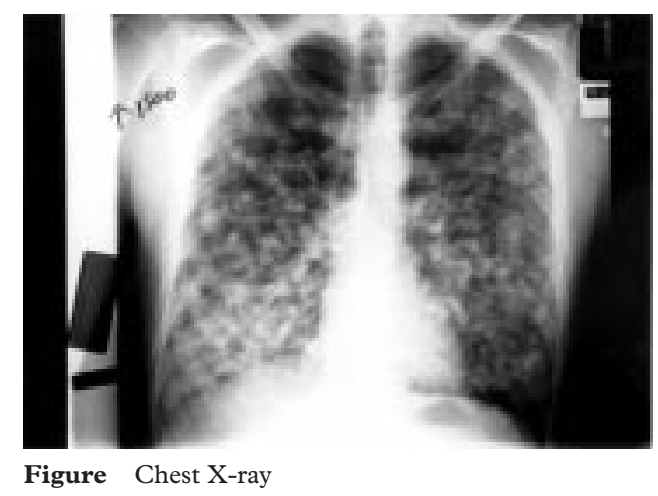

\section{Questions}

1 What is the differential diagnosis?

2 What treatment would you advise?

3 What are the risk factors and other complications associated with this condition? 
Answers

QUESTION 1

There are many causes of diffuse nodular shadowing on chest X-ray (box 1). The patient's rash was suggestive of chicken-pox. His three sons also had this diagnosis. A diagnosis of presumptive varicella pneumonia was based on the patient's response to intravenous acyclovir and laboratory findings which included negative findings for tuberculosis, legionella, cryptococcosis, and pneumocystis carinii pneumonitis. Blood and sputum cultures and sputum cytology were also negative. The patient refused fiberoptic bronchoscopy. After 7 days of treatment with acyclovir, his condition greatly improved and he was discharged.

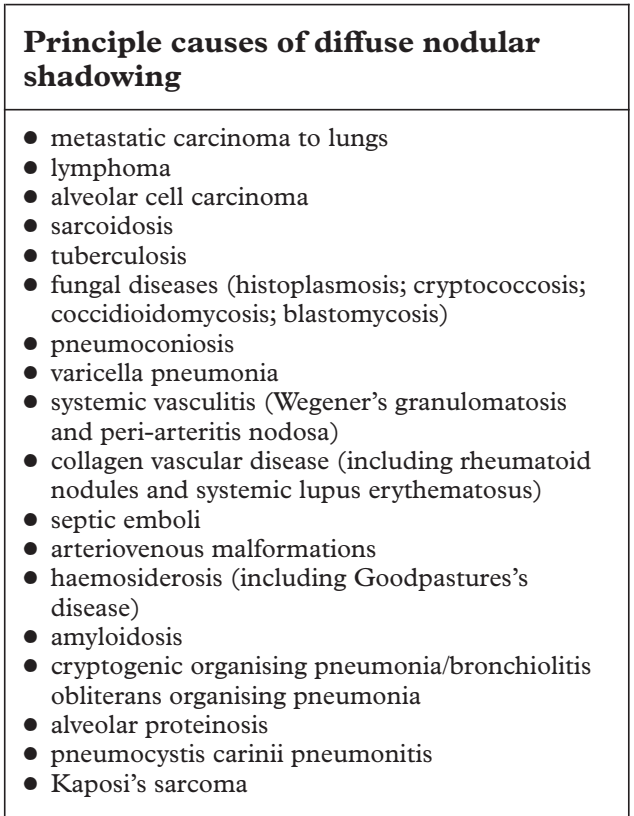

Box 1

QUESTION 2

Acyclovir is preferred over vidarabine for treatment. It has been more effective in shortening the cutaneous course of varicella infection in the immunocompromised patient. Other advantages of acyclovir are reduced haematopoietic and neurological toxic reactions, shorter infusion time, and increased solubility. Vidarabine and foscarnet have also been used to treat varicella infection, but with much less success. The recommended doses of these drugs are listed in box 2 .

QUESTION 3

The identification of several risk factors for adult varicella pneumonia is of special interest for possible chemoprophylaxis (box 3). Reports have show an increased risk for varicella pneumonia in patients after prolonged treatment with corticosteroids, cytotoxic or radiation therapy. In addition, it has been reported that

\begin{tabular}{|l|}
\hline Antiviral treatment for varicella \\
infections \\
\hline Varicella pneumonitis \\
- acyclovir, iv $30 \mathrm{mg} / \mathrm{kg} /$ day every $8 \mathrm{~h}$ or \\
- vidarabine, iv $10 \mathrm{mg} / \mathrm{kg} /$ day every $12 \mathrm{~h}$ or \\
(duration depends on clinical course) \\
Varicella in the immunocompromised host \\
- acyclovir, iv $30 \mathrm{mg} / \mathrm{kg} /$ day every $8 \mathrm{~h}$ for $5-7$ days \\
or \\
- vidarabine, iv $10 \mathrm{mg} / \mathrm{kg} /$ day every $12 \mathrm{~h}$ for $5-7$ \\
- days or \\
- foscarnet, iv $40 \mathrm{mg} / \mathrm{kg} /$ day every $12 \mathrm{~h}$ for $5-7$ \\
days \\
- acycella in the immunocompetent host \\
Box 2 \\
- cho $800 \mathrm{mg}$ five times a day for 5 days \\
Risk factors for adult varicella \\
pneumonia \\
- pregnancy \\
- immunosuppressed individuals \\
- older individuals \\
- chere cutaneous $/ \mathrm{haemorr}$ obstructive pulmonary disease
\end{tabular}

Box 3

conventional 'low-dose' corticosteroid therapy ( $<2 \mathrm{mg} / \mathrm{kg}$ or $5-20 \mathrm{mg}$ daily), and short-term steroid therapy for acute asthma attacks may predispose patients to disseminated varicella. ${ }^{1}$

The US Food and Drug Administration have warned that patients on immunosuppressant doses of corticosteroids should be warned to avoid exposure to chicken pox and, if exposed, obtain medical advice. The UK Committee on Safety of Medicines has recently recommended that all patients taking systemic corticosteroids for purposes other than replacement should be regarded as at risk of severe varicella zoster unless there is a history of previous chicken pox. Even then, a clinical history of chicken pox is not necessarily reliable, and testing of all such patient to identify the seronegative cases at risk might be wise, albeit expensive. ${ }^{2}$ The Joint Committee on Vaccination and Immunization has recommended that immunosuppressed patients who have been receiving steroids at a daily dose of $2 \mathrm{mg} / \mathrm{kg}$ for more than a week within 3 months of direct contact with chicken pox should be passively immunized with varicella zoster immunoglobins if they do not already have antibodies to varicella zoster. Similar recommendations seem appropriate with other immunosuppressive agents, especially when several agents are combined.

The only identifiable risk factors for this patient was that he had smoked 50 cigarettes/ day for the past 10 years and he had a severe cutaneous disease. Further investigation revealed him to be HIV positive with a CD4 


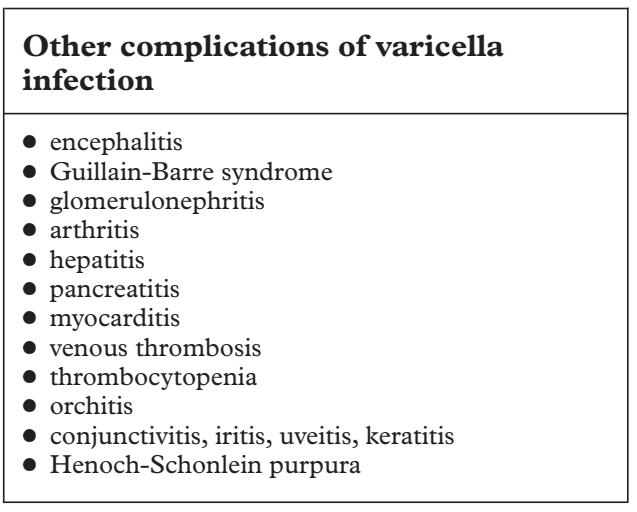

Box 4

count of 0 . He denied any history of intravenous drug use or high-risk sexual behaviour. Eight months later, this patient developed varicella retinopathy and progressive outer retinal necrosis (PORN syndrome), which resulted in permanent visual loss. PORN is a distinct form of necrotizing herpetic retinopathy that has been reported with increasing frequency in AIDS patients. Varicella zoster virus is currently the only agent that has been associated with the disease.

Various complications of varicella infection may occur (box 4). The most common and serious complication is varicella pneumonia.

\section{Discussion}

Varicella is usually a benign childhood disease, while in the adult it may be complicated by pneumonia with high morbidity and mortality rates. Varicella is one of the most common transmittable diseases. However less than 2\%

1 Feldman S. Varicella-Zoster virus pneumonitis. Chest 1994;106:22S-27S.

2 Weir WRC. Commentary: severe varicella/zoster and adenovirus infections in immunocompromised patients. Thorax 1995;50:1222-3.

3 Hockberger RS, Rothstein RJ. Varicella pneumonia in adults: a spectrum of disease. Ann Emerg Med 1986;15:931-4. of 3-4 million annual cases in the US occur in adults. The most common complications of adult varicella are pulmonary manifestations, especially pneumonia (incidence rate $10-50 \%) .^{3}$ Ninety per cent of pulmonary manifestations of varicella occur after the age of 19 years, mainly in the third decade. Untreated adult varicella pneumonia is fatal in approximately $10 \%$ of cases. ${ }^{4}$

Pneumonia occurs early in the course of varicella, within 1-6 days after the onset of the rash. Varicella pneumonia should be considered in any adult with chicken pox who complains of dyspnoea, cough and fever. Physical findings usually correlate poorly with the degree of pneumonia.

Chest X-rays of varicella pneumonia can vary; abnormal findings are seen in about $16 \%$ of cases with primary varicella. ${ }^{5}$ Findings may include a patchy, diffuse air-space consolidation or diffuse interstitial infiltrates that may be nodular, with the severity correlating with the diffuseness of the rash. The infiltrate can be associated with a proteinaceous exudate, oedema and hyaline membrane formation which can progress to respiratory distress syndrome. In the later stages, diffuse pulmonary microcalcifications of $2-3 \mathrm{~mm}$ in diameter have been described. ${ }^{5}$

Aggressive and early treatment with acyclovir seems to prevent the potentially catastrophic consequences of varicella pneumonia. Oral acyclovir chemoprophylaxis may have some benefit in high-risk populations with chicken pox.

\section{Final diagnosis}

Varicella pneumonia.

Keywords: chicken pox; varicella pneumonia; acyclovir

\footnotetext{
4 Triebwasser JH, Harris RE, Bryant RE, Rhoades ER. Varicella pneumonia in adults: report of seven cases and a review of literature. Medicine 1967;46:406-23.

5 Gogos CA, Bassaris HP, Vagenakis AG. Varicella pneumonia in adults. A review of pulmonary manifestations, risk factors and treatment. Respiration 1992;59:339-43.
} 